\title{
Association between regulating synaptic membrane exocytosis 2 gene polymorphisms and degenerative lumbar scoliosis
}

\author{
KI-TACK KIM ${ }^{1 *}$, JONG SEOK LEE ${ }^{2 *}$, BYOUNG WOOK LEE ${ }^{3}$, HOSIK SEOK $^{4}$, \\ HYE SOOK JEON ${ }^{4}$, JUN HO KIM ${ }^{4}$ and JOO-HO CHUNG ${ }^{4}$ \\ ${ }^{1}$ Department of Orthopedic Surgery, Spine Center, Kyung Hee University East-West Neo Medical Center, Kangdong-gu, \\ Seoul 134-090; Departments of ${ }^{2}$ Emergency Medicine and ${ }^{3}$ Biochemistry and Molecular Biology, \\ Kyung Hee University; ${ }^{4}$ Kohwang Medical Research Institute, School of Medicine, \\ Kyung Hee University, Dongdaemun-gu, Seoul 130-701, Republic of Korea
}

Received February 28, 2013; Accepted April 18, 2013

DOI: $10.3892 /$ br.2013.101

\begin{abstract}
Degenerative lumbar scoliosis (DLS) is a spinal deformity that develops after skeletal maturity and progresses with age. In contrast to adolescent idiopathic scoliosis, the genetic association of DLS has not yet been elucidated. The purpose of this study was to investigate the association between regulating synaptic membrane exocytosis 2 (RIMS2, OBOE) gene polymorphisms and DLS. Two coding single-nucleotide polymorphisms [rs2028945 (Gln1200Gln) and rs10461 (Ala1327Ala)] of RIMS2 were selected and genotyped by direct sequencing. As a result, the rs10461 was associated with DLS in allele frequencies $(\mathrm{P}=0.008)$ and genotype distributions $(\mathrm{P}=0.006$ in the codominant model, 0.018 in the dominant model and 0.029 in the recessive model). In the analysis of haplotypes, two haplotypes exhibited significant differences between the control and DLS groups (CC haplotype, $\mathrm{P}=0.009$ in the codominant model, 0.038 in the dominant model and 0.030 in the recessive model; CT haplotype, $\mathrm{P}=0.041$ in the codominant model and 0.021 in the dominant model). These findings suggest that RIMS2 may be associated with the development of DLS.
\end{abstract}

Correspondence to: Professor Joo-Ho Chung, Kohwang Medical Research Institute, School of Medicine, Kyung Hee University, 1 Hoegi-dong, Dongdaemun-gu, Seoul 130-701, Republic of Korea E-mail: jhchung@khu.ac.kr

*Contributed equally

Abbreviations: RIMS2, regulating synaptic membrane exocytosis 2; SNP, single-nucleotide polymorphism; HWE, Hardy-Weinberg equilibrium; LD, linkage disequilibrium; DLS, degenerative lumbar scoliosis

Key words: degenerative lumbar scoliosis, regulating synaptic membrane exocytosis 2 , polymorphism

\section{Introduction}

Degenerative lumbar scoliosis (DLS) is defined as a spinal deformity with a Cobb angle of $>10^{\circ}$, which develops after skeletal maturity without a previous history of scoliosis. It generally presents in the lumbar or thoracolumbar part of the spine and may be associated with severe back pain $(1,2)$. The prevalence of DLS increases with age and its incidence in patients $>50$ years of age was reported to be $\sim 6 \%$ (3). The etiology of DLS has not been clearly established. However, the most commonly proposed causes of DLS were ost eoporosis and degenerative diseases of the spinal column (3). Several factors, including life-style, intrinsic mediators and hormonal or genetic factors, are likely to affect the development or the acceleration of DLS $(4,5)$.

Rab3-interacting molecules (RIMs) are presynaptic active zone proteins that perform an essential function in neurotransmitter release $(6,7)$. RIMs were identified as putative effectors for Rab3, which is a synaptic vesicle protein that regulates neurotransmitter release $(6,7)$. RIMs have been shown to interact with multiple synaptic proteins, such as UNC-13 homolog B (C. elegans) (UNC13B, Munc13) (8), ELKS/Rab6-interacting/CAST family member 1 (ERC1, ELKS) (9,10), RIM-binding proteins (RIM-BPs) (11), $\alpha$-liprins and synaptotagmin 1 (12-14). RIMs are encoded by four regulating synaptic membrane exocytosis genes (RIMS1-4), of which RIMS1, RIMS3 and RIMS4 express a single isoform (RIM1 $\alpha, 3 \gamma$ and $4 \gamma$, respectively), whereas RIMS2 (RAB3IP3, OBOE) expresses three isoforms (RIM2 $\alpha$, RIM $2 \beta$ and RIM $2 \gamma$ ) via independent internal promoters (15).

Two single-nucleotide polymorphisms (SNPs) [rs2028945 (Gln1200Gln) and rs10461 (Ala1327Ala)] in the coding region of RIMS2 in DLS were investigated in this study.

\section{Subjects and methods}

Subjects and clinical phenotypes. A total of 51 DLS patients and 145 control subjects were enrolled in this study. DLS patients were recruited from the Spine Center of Kyung Hee University East-West Neo Medical Center (Seoul, Korea) and the National Medical Center (Seoul, Korea) and were selected 
among $\sim 10,000$ patients per year during a 3 -year period. Each patient was diagnosed by a specialized spinal surgeon and fulfilled all physical examination and radiographic criteria. The DLS group comprised 44 females and 7 males and the mean age was $68.67 \pm 8.00$ years [mean \pm standard deviation (SD)]. The Cobb angle in the DLS group was $19.36 \pm 7.38^{\circ}$ and the lateral listhesis was $4.84 \pm 4.24 \mathrm{~mm}$. A left convex curve was observed more frequently compared to a right convex curve (31 and 20 patients, respectively) (Table I). DLS patients were divided into two subgroups according to clinical characteristics, including Cobb angle $\left(\leq 30^{\circ}\right.$ vs. $\left.>30^{\circ}\right)$, lateral listhesis ( $\leq 5 \mathrm{vs} .>5 \mathrm{~mm}$ ) and curve direction (left vs. right) $(16,17)$. The control group included 145 patients (124 females and 21 males) who were recruited from a general health check-up program after it was confirmed that they had no clinical evidence of scoliosis or any other severe disorders. The gender and age of the control group were matched with those of the DLS group. This study was conducted according to the Declaration of Helsinki guidelines. Written informed consent was obtained from each individual. The research protocol was approved by the Ethics Review Committee of the Medical Research Institute, Kyung Hee University Medical Center, Seoul, Korea.

SNP selection and genotyping. We investigated SNPs in the coding region of RIMS2. The related information was obtained from the SNP database (www.ncbi.nlm.nih.gov/SNP, dbSNP BUILD131). The SNPs with unknown heterozygosity, minor allele frequencies $<10 \%$ and no data regarding Asian populations (rs17854256, rs76323676 and rs61753732) were excluded. Consequently, rs2028945 (Gln1200Gln) and rs10461 (Ala1327Ala) were selected for this investigation. Genomic DNA was extracted from the blood sample of each subject using Qiagen DNA Extraction kit (Qiagen, Tokyo, Japan) following the manufacturer's instructions and was amplified using the primers for each SNP of RIMS2 (Table II). PCR products were sequenced by an ABI PRISM 3730xl DNA Analyzer (Applied Biosystems Inc., Foster City, CA, USA). Sequence data were analyzed using SeqMan II software (DNASTAR, Inc., Madison, WI, USA).

Statistical analysis. The Hardy-Weinberg equilibrium (HWE) was assessed using SNPStats (http://bioinfo.iconcologia.net/ index.php) (18). The allele frequencies of each SNP were compared by the Pearson's Chi-square test. The effect of SNP genotypes was analyzed using the codominant, dominant and recessive models. Logistic regression analysis was used to calculate the odds ratio (ORs), 95\% confidence intervals (CIs) and P-values with controlling age and gender as covariables. The linkage disequilibrium (LD) was assessed using Haploview software version 4.1 (Broad Institute, Cambridge, MA, USA). LD block was constructed using the Gabriel method $(19,20)$. The association of SNPs and haplotypes was analyzed using SNPStats, HapAnalyzer version 1.0 (http://hap. ngri.go.kr/), and HelixTree (Golden Helix Inc., Bozeman, MT, USA) software.

Statistical analysis was performed using the package of SPSS Statistics software version 17.0 (SPSS Inc., Chicago, IL, USA). $\mathrm{P}<0.05$ was considered to indicate a statistically significant difference.
Table I. Demographic characteristics of the study subjects.

\begin{tabular}{lcc}
\hline Characteristic & Control group & DLS group \\
\hline Number of subjects (n) & 145 & 51 \\
Male/female (n) & $124 / 21$ & $44 / 7$ \\
Age (mean \pm SD, years) & $65.57 \pm 8.65$ & $68.67 \pm 8.00$ \\
Cobb angle (degrees) & & $19.36 \pm 7.38$ \\
Lateral listhesis (mm) & & $4.84 \pm 4.24$ \\
Curve direction (left/right) & & $31 / 20$ \\
\hline
\end{tabular}

DLS, degenerative lumbar scoliosis; SD, standard deviation.
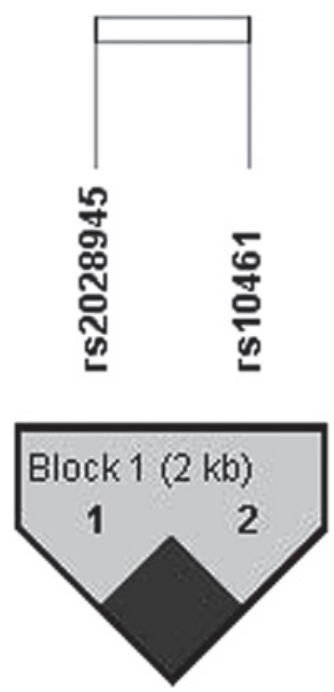

Figure 1. Linkage disequilibrium block between rs2028945 and rs10461 in regulating synaptic membrane exocytosis 2 (RIMS2).

\section{Results}

The clinical characteristics of control subjects and DLS patients are summarized in Table I. The mean age of the control and the DLS group was $65.57 \pm 8.65$ (mean $\pm \mathrm{SD}$ ) and $68.67 \pm 8.00$ years, respectively. In the DLS group, the average Cobb angle was $19.36 \pm 7.38^{\circ}$ and the length of the lateral listhesis was $4.84 \pm 4.24 \mathrm{~mm}$. Patients with a left convex curve outnumbered those with a right convex curve (31 and 20, respectively) (Table I).

The genotype distributions of rs2028945 and rs10461 were in HWE equilibrium $(\mathrm{P}>0.05)$. In an analysis of the allele frequencies on two SNPs, the C allele of rs10461 was more frequently encountered in the DLS group compared to the control group (OR=1.857; 95\% CI, 1.174-2.937 and $\mathrm{P}=0.008$ ) (Table III). In an analysis of the genotype distributions, the rs10461 was associated with DLS (OR=1.93; 95\% CI, $1.20-3.10$ and $\mathrm{P}=0.006$ in the codominant model; $\mathrm{OR}=2.62 ; 95 \% \mathrm{CI}, 1.12-6.13$ and $\mathrm{P}=0.018$ in the dominant model, and $\mathrm{OR}=2.25 ; 95 \% \mathrm{CI}, 1.10-4.61$ and $\mathrm{P}=0.029$ in the recessive model). The rs 2028945 was not significantly different between the control and the DLS groups (Table IV). One LD block was constructed between rs2028945 and rs10461 with the Gabriel method (Fig. 1). Of the haplotypes in the block, the 
Table II. Primer sequences used for each SNP in RIMS2.

\begin{tabular}{|c|c|c|}
\hline SNP & Sequence $\left(5^{\prime}-3^{\prime}\right)$ & Product size (bp) \\
\hline \multicolumn{3}{|l|}{ rs2028945 } \\
\hline Sense & TCCTCACTGAACACTCATTCAGG & 543 \\
\hline Antisense & CTCAGCCCAGTGGAATCTTTAAC & \\
\hline \multicolumn{3}{|l|}{ rs10461 } \\
\hline Sense & AGATCATCGTCTGGGGAGATTA & 343 \\
\hline Antisense & CCCTAGAAACAGGCTCAGAAGA & \\
\hline
\end{tabular}

SNP, single-nucleotide polymorphism; RIMS2, regulating synaptic membrane exocytosis 2.

Table III. Genotype and allele frequencies of RIMS2 polymorphisms in the control and DLS groups.

\begin{tabular}{|c|c|c|c|c|c|c|c|c|}
\hline \multirow[b]{2}{*}{ SNP } & \multirow[b]{2}{*}{ Type } & \multicolumn{2}{|c|}{ Control } & \multicolumn{2}{|c|}{ DLS } & \multirow[b]{2}{*}{ Model } & \multirow[b]{2}{*}{ OR $(95 \% \mathrm{CI})$} & \multirow[b]{2}{*}{ P-value } \\
\hline & & No. & $(\%)$ & No. & $(\%)$ & & & \\
\hline rs2028945 & Genotype & & & & & & & \\
\hline \multirow[t]{6}{*}{ Gln1200Gln } & $\mathrm{C} / \mathrm{C}$ & 49 & 33.8 & 20 & 39.2 & Codominant & $0.72(0.45-1.16)$ & 0.18 \\
\hline & $\mathrm{C} / \mathrm{T}$ & 69 & 47.6 & 26 & 51.0 & Dominant & $0.79(0.40-1.53)$ & 0.48 \\
\hline & $\mathrm{T} / \mathrm{T}$ & 27 & 18.6 & 5 & 9.8 & Recessive & $0.44(0.16-1.23)$ & 0.09 \\
\hline & Allele & & & & & & & \\
\hline & $\mathrm{C}$ & 167 & 57.6 & 66 & 64.7 & & 1 & \\
\hline & $\mathrm{T}$ & 123 & 42.4 & 36 & 35.3 & & $0.74(0.46-1.18)$ & 0.21 \\
\hline rs10461 & Genotype & & & & & & & \\
\hline \multirow[t]{6}{*}{ Ala1327Ala } & $\mathrm{T} / \mathrm{T}$ & 45 & 31.0 & 8 & 15.7 & Codominant & $1.93(1.20-3.10)$ & 0.006 \\
\hline & $\mathrm{T} / \mathrm{C}$ & 71 & 49.0 & 25 & 49.0 & Dominant & $2.62(1.12-6.13)$ & 0.018 \\
\hline & $\mathrm{C} / \mathrm{C}$ & 29 & 20.0 & 18 & 35.3 & Recessive & $2.25(1.10-4.61)$ & 0.029 \\
\hline & Allele & & & & & & & \\
\hline & $\mathrm{T}$ & 161 & 55.5 & 41 & 40.2 & & 1 & \\
\hline & $\mathrm{C}$ & 129 & 44.5 & 61 & 59.8 & & $1.857(1.174-2.937)$ & 0.008 \\
\hline
\end{tabular}

P-values were calculated by logistic regression analysis following adjustment for age and gender. Bold numbers indicate significant association. RIMS2, regulating synaptic membrane exocytosis 2; SNP, single-nucleotide polymorphism; DLS, degenerative lumbar scoliosis; OR, odds ratio; CI, confidence interval.

CC and CT haplotypes were associated with the risk of DLS (CC haplotype: $\mathrm{P}=0.009$ in the codominant model, $\mathrm{P}=0.038$ in the dominant model and $\mathrm{P}=0.030$ in the recessive model; CT haplotype: $\mathrm{P}=0.041$ in the codominant model and $\mathrm{P}=0.021$ in the dominant model) (Table IV).

The results obtained in this study were compared with different ethnic populations by searching the human SNP database (www.ncbi.nlm.nih.gov/SNP; dbSNP BUILD 131), which includes the genotype frequencies of each SNP. Genotype distributions in our control subjects were similar to those in Asian populations, particularly the Chinese population (Table V).

\section{Discussion}

DLS develops de novo in adulthood and presents more frequently in the elderly (3). With increasing life expectancy,
DLS may complicate degenerative spondylolisthesis, lateral listhesis or spinal stenosis, in which the neural elements are compressed by bone and soft tissue, leading to nerve root ischemia (2). Therefore, patients with accelerated DLS may develop severe back pain and neurological deficits (3). Despite a variety of studies on DLS, the etiology remains unknown. Therefore, the potential of RIMS2 as a candidate gene was evaluated. Our results demonstrated that rs10461 (Ala1327Ala) was significantly associated with DLS. It was hypothesized that the $\mathrm{C}$ allele of rs10461 may be a risk factor in the development of DLS (Table III). In addition, two haplotypes (CC and $\mathrm{CT}$ ) exhibited significant differences between the control and DLS groups. The results indicated that RIMS2 may be associated with DLS. However, RIMS2 polymorphisms were not associated with the clinical features of DLS (Cobb angle, lateral listhesis and curve direction) (data not shown). 
Table IV. Haplotype distributions of RIMS2 polymorphisms (rs2028945 and rs 10461) in the control and DLS groups.

\begin{tabular}{|c|c|c|c|c|c|c|c|c|c|}
\hline \multirow[b]{2}{*}{ Haplotype } & \multicolumn{3}{|c|}{ Control } & \multicolumn{3}{|c|}{ DLS } & \multirow[b]{2}{*}{ Model } & \multirow[b]{2}{*}{ OR $(95 \%$ CI $)$} & \multirow[b]{2}{*}{ P-value } \\
\hline & No. & $(\%)$ & Freq & No. & $(\%)$ & Freq & & & \\
\hline \multicolumn{10}{|c|}{ HAP1 (CC) } \\
\hline $\mathrm{HH}$ & 29 & 20.0 & \multirow{3}{*}{0.44} & 29 & 20.0 & \multirow{3}{*}{0.60} & Codominant & $1.86(1.16-2.96)$ & 0.009 \\
\hline $\mathrm{H}-$ & 71 & 49.0 & & 71 & 49.0 & & Dominant & $2.42(1.05-5.56)$ & 0.038 \\
\hline-- & 45 & 31.0 & & 45 & 31.0 & & Recessive & $2.18(1.08-4.41)$ & 0.030 \\
\hline \multicolumn{10}{|c|}{ HAP2 (TT) } \\
\hline $\mathrm{HH}$ & 27 & 18.6 & \multirow{3}{*}{0.42} & 27 & 18.6 & \multirow{3}{*}{0.35} & Codominant & $0.74(0.46-1.18)$ & 0.208 \\
\hline $\mathrm{H}-$ & 69 & 47.6 & & 69 & 47.6 & & Dominant & $0.79(0.41-1.53)$ & 0.486 \\
\hline-- & 49 & 33.8 & & 49 & 33.8 & & Recessive & $0.48(0.17-1.31)$ & 0.150 \\
\hline \multicolumn{10}{|l|}{ HAP3 (CT) } \\
\hline $\mathrm{HH}$ & 4 & 2.8 & \multirow{3}{*}{0.13} & 4 & 2.8 & \multirow{3}{*}{0.05} & Codominant & $0.38(0.15-0.96)$ & 0.041 \\
\hline $\mathrm{H}-$ & 30 & 20.7 & & 30 & 20.7 & & Dominant & $0.28(0.09-0.83)$ & 0.021 \\
\hline-- & 111 & 76.6 & & 111 & 76.6 & & Recessive & $0.70(0.08-6.46)$ & 0.757 \\
\hline
\end{tabular}

P-values were calculated by logistic regression analysis following adjustment for age and gender. Bold numbers indicate significant association. RIMS2, regulating synaptic membrane exocytosis 2; SNP, single-nucleotide polymorphism; DLS, degenerative lumbar scoliosis; Freq, frequency; OR, odds ratio; CI, confidence interval.

Table V. Genotype distributions of RIMS2 polymorphisms among ethnic populations.

\begin{tabular}{|c|c|c|c|c|c|}
\hline \multirow[b]{2}{*}{ SNP } & \multicolumn{5}{|c|}{ Populations } \\
\hline & Korean & European & Chinese & Japanese & Sub-Saharan African \\
\hline \multicolumn{6}{|c|}{ rs2028945 } \\
\hline $\mathrm{C} / \mathrm{C}$ & 0.338 & 0.833 & 0.267 & 0.182 & 0.850 \\
\hline $\mathrm{C} / \mathrm{T}$ & 0.476 & 0.167 & 0.489 & 0.341 & 0.133 \\
\hline $\mathrm{T} / \mathrm{T}$ & 0.186 & - & 0.244 & 0.477 & 0.017 \\
\hline \multicolumn{6}{|c|}{ rs10461 } \\
\hline $\mathrm{C} / \mathrm{C}$ & 0.200 & 0.414 & 0.222 & 0.159 & 0.153 \\
\hline $\mathrm{C} / \mathrm{T}$ & 0.490 & 0.483 & 0.356 & 0.341 & 0.610 \\
\hline $\mathrm{T} / \mathrm{T}$ & 0.103 & 0.103 & 0.422 & 0.500 & 0.237 \\
\hline
\end{tabular}

From dbSNPBUILD131 (www.ncbi.nlm.nih.gov/SNP). RIMS2, regulating synaptic membrane exocytosis 2; SNP, single-nucleotide polymorphism.

RIMS comprise four members, RIMS1-4. RIMS1 and RIMS2 are 512.7 and $747.9 \mathrm{~kb}$ in size, respectively, whereas RIMS3 and RIMS4 are 15.4 and $54.5 \mathrm{~kb}$, respectively $(8,15)$. RIMS1 expresses RIM1 $\alpha$; the gene may encode a single isoform. By contrast, RIMS2 encodes three isoforms (RIM $2 \alpha$, RIM2 $\beta$ and RIM2 $\gamma$ ); besides the exon for the $\alpha$ isoform, two additional separate exons in RIMS2 may encode the $\mathrm{N}$-termini of RIM $2 \beta$ and RIM $2 \gamma$ through $\beta$ - and $\gamma$-specific promoters, respectively. RIMS3 and RIMS4 express a single isoform, RIM $3 \gamma$ and RIM $4 \gamma$, respectively $(8,15)$. The $\alpha$-RIMs (RIM1 $\alpha$ and RIM2 $\alpha$ ) contain the full complement of domains which are the $\mathrm{N}$-terminal $\mathrm{Zn}^{2+}$-finger domain, the central PDZ and $\mathrm{C}_{2} \mathrm{~A}$ domains and the $\mathrm{C}$-terminal $\mathrm{C}_{2} \mathrm{~B}$ domain (15). They are able to bind to Rab3 and Munc13 proteins $(13,21,22)$ and closely interact with $\alpha$-liprins (13), which in turn regulate the active zone structure $(23,24)$. Therefore, $\alpha$-RIMs are considered to be essential for regulating neurotransmitter releases $(8,13,25,26)$, some of which are involved in the neurobiology of schizophrenia $(27,28)$.

The exocytosis of neurotransmitter-filled synaptic vesicles is under tight regulation in presynaptic nerve terminals. RIMs may mediate the regulation of exocytosis via interacting with multiple synaptic proteins, such as Rab3 $(6,7)$, Munc13 (8), ELKS $(9,10)$, RIM-BPs (11), $\alpha$-liprins and synaptotagmin 1 (12-14). In addition, RIMs are indirectly connected with the active zone proteins Piccolo and Bassoon via ELKS (29). In a previous study, the experimental loss of $\alpha$-RIMs resulted in the severe impairment of motor control, profound defects of synaptic transmission at neuromuscular junctions and increment of irregularly distributed motor synapses in skeletal muscle fibers (8). These results suggested 
that RIMs may affect the function of the neuromuscular system. However, no published studies assessing the potential role of RIMs in bone degeneration or the genetic association of RIMS polymorphisms in DLS are available. We demonstrated that RIMS2, known as a regulatory gene for synaptic membrane exocytosis in the central nervous system, may be a candidate gene associated with the risk of DLS in the Korean population. However, additional studies including a larger number of subjects and different populations are required to confirm these findings. In conclusion, the rs10461 in RIMS2 was associated with DLS in the Korean population. This finding suggests that RIMS2 may affect the development of DLS.

\section{References}

1. Aebi M: The adult scoliosis. Eur Spine J 14: 925-948, 2005.

2. Ploumis A, Transfledt EE and Denis F: Degenerative lumbar scoliosis associated with spinal stenosis. Spine J 7: 428-436, 2007.

3. Oskouian RJ Jr and Shaffrey CI: Degenerative lumbar scoliosis. Neurosurg Clin N Am 17: 299-315, 2006.

4. Ferrari S, Rizzoli R and Bonjour JP: Heritable and nutritional influences on bone mineral mass. Aging (Milano) 10: 205-213, 1998.

5. Kawaguchi Y, Kanamori M, Ishihara H, Ohmori K, Matsui H and Kimura T: The association of lumbar disc disease with vitamin-D receptor gene polymorphism. J Bone Joint Surg Am 84-A: 2022-2028, 2002.

6. Dresbach T, Qualmann B, Kessels MM, Garner CC and Gundelfinger ED: The presynaptic cytomatrix of brain synapses Cell Mol Life Sci 58: 94-116, 2001.

7. Lonart G: RIM1: an edge for presynaptic plasticity. Trends Neurosci 25: 329-332, 2002.

8. Schoch S, Mittelstaedt T, Kaeser PS, et al: Redundant functions of RIM1 $\alpha$ and RIM $2 \alpha$ in $\mathrm{Ca}^{2+}$-triggered neurotransmitter release. EMBO J 25: 5852-5863, 2006.

9. Ohtsuka T, Takao-Rikitsu E, Inoue E, et al: Cast: a novel protein of the cytomatrix at the active zone of synapses that forms a ternary complex with RIM1 and munc13-1. J Cell Biol 158: 577-590, 2002

10. Wang Y, Liu X, Biederer T and Südhof TC: A family of RIM-binding proteins regulated by alternative splicing: implications for the genesis of synaptic active zones. Proc Natl Acad Sci USA 99: 14464-14469, 2002.

11. Wang Y, Sugita S and Südhof TC: The RIM/NIM family of neuronal $\mathrm{C}_{2}$ domain proteins. Interactions with $\mathrm{Rab3}$ and a new class of Src homology 3 domain proteins. J Biol Chem 275: 20033-20044, 2000

12. Coppola T, Magnin-Luthi S, Perret-Menoud V, et al: Direct interaction of the Rab3 effector RIM with $\mathrm{Ca}^{2+}$ channels, SNAP-25, and synaptotagmin. J Biol Chem 276: 32756-32762, 2001.
13. Schoch S, Castillo PE, Jo T, et al: RIM1alpha forms a protein scaffold for regulating neurotransmitter release at the active zone. Nature 415: 321-326, 2002.

14. Schoch S and Gundelfinger ED: Molecular organization of the presynaptic active zone. Cell Tissue Res 326: 379-391, 2006.

15. Wang Y and Südhof TC: Genomic definition of RIM proteins: evolutionary amplification of a family of synaptic regulatory proteins. Genomics 81: 126-137, 2003.

16. Pritchett JW and Bortel DT: Degenerative symptomatic lumbar scoliosis. Spine (Phila Pa 1976) 18: 700-703, 1993.

17. Chin KR, Furey $\mathrm{C}$ and Bohlman HH: Risk of progression in de novo low-magnitude degenerative lumbar curves: natural history and literature review. Am J Orthop (Belle Mead NJ) 38: 404-409, 2009

18. Solé X, Guinó E, Valls J, Iniesta R and Moreno V: SNPStats: a web tool for the analysis of association studies. Bioinformatics 22: 1928-1929, 2006

19. Gabriel SB, Schaffner SF, Nguyen H, et al: The structure of haplotype blocks in the human genome. Science 296: 2225-2229, 2002.

20. Barrett JC, Fry B, Maller J and Daly MJ: Haploview: analysis and visualization of LD and haplotype maps. Bioinformatics 21 : 263-265, 2005.

21. Betz A, Thakur P, Junge HJ, et al: Functional interaction of the active zone proteins Munc13-1 and RIM1 in synaptic vesicle priming. Neuron 30: 183-196, 2001.

22. Wang X, Hu B, Zimmermann B and Kilimann MW: Rim1 and rabphilin-3 bind Rab3-GTP by composite determinants partially related through N-terminal alpha-helix motifs. J Biol Chem 276: 32480-32488, 2001.

23. Zhen $M$ and Jin Y: The liprin protein SYD-2 regulates the differentiation of presynaptic termini in C.elegans. Nature 401: 371-375, 1999.

24. Kaufmann N, DeProto J, Ranjan R, et al: Drosophila liprin-alpha and the receptor phosphatase Dlar control synapse morphogenesis. Neuron 34: 27-38, 2002.

25. Koushika SP, Richmond JE, Hadwiger G, et al: A post-docking role for active zone protein Rim. Nat Neurosci 4: 997-1005, 2001.

26. Castillo PE, Schoch S, Schmitz F, et al: RIM1alpha is required for presynaptic long-term potentiation. Nature 415: 327-330, 2002 .

27. Weidenhofer J, Bowden NA, Scott RJ and Tooney PA: Altered gene expression in the amygdala in schizophrenia: up-regulation of genes located in the cytomatrix active zone. Mol Cell Neurosci 31: 243-250, 2006.

28. Weidenhofer J, Scott RJ and Tooney PA: Investigation of the expression of genes affecting cytomatrix active zone function in the amygdala in schizophrenia: effects of antipsychotic drugs. J Psychiatr Res 43: 282-290, 2009.

29. Takao-Rikitsu E, Mochida S, Inoue E, et al: Physical and functional interaction of the active zone proteins, CAST, RIM1, and Bassoon, in neurotransmitter release. J Cell Biol 164: 301-311, 2004. 